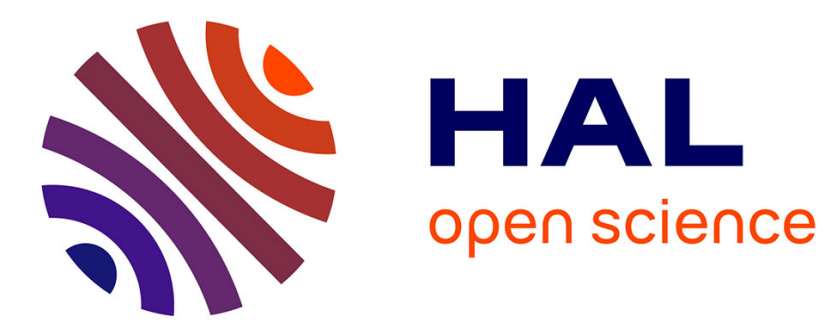

\title{
Uncertainty quantification for the Modal Phase Collinearity of complex mode shapes
} Szymon Gres, Michael Döhler, Palle Andersen, Laurent Mevel

\section{To cite this version:}

Szymon Gres, Michael Döhler, Palle Andersen, Laurent Mevel. Uncertainty quantification for the Modal Phase Collinearity of complex mode shapes. Mechanical Systems and Signal Processing, 2021, 152, pp.107436. 10.1016/j.ymssp.2020.107436 . hal-03276212

\section{HAL Id: hal-03276212 \\ https://hal.inria.fr/hal-03276212}

Submitted on 1 Jul 2021

HAL is a multi-disciplinary open access archive for the deposit and dissemination of scientific research documents, whether they are published or not. The documents may come from teaching and research institutions in France or abroad, or from public or private research centers.
L'archive ouverte pluridisciplinaire HAL, est destinée au dépôt et à la diffusion de documents scientifiques de niveau recherche, publiés ou non, émanant des établissements d'enseignement et de recherche français ou étrangers, des laboratoires publics ou privés. 


\title{
Uncertainty quantification for the Modal Phase Collinearity of complex mode shapes ${ }^{\text {th }}$
}

\author{
Szymon Greśs,*, Michael Döhler ${ }^{\mathrm{b}}$, Palle Andersen ${ }^{\mathrm{c}}$, Laurent Mevel ${ }^{\mathrm{b}}$ \\ ${ }^{a}$ Aalborg University, Department of Civil and Structural Engineering, Thomas Manns Vej 23, 9000 Aalborg, Denmark \\ ${ }^{b}$ Univ. Gustave Eiffel, Inria, COSYS-SII, I4S, Campus de Beaulieu, 35042 Rennes, France \\ ${ }^{c}$ Structural Vibration Solutions A/S, NOVI Science Park, 9220 Aalborg, Denmark
}

\begin{abstract}
The Modal Phase Collinearity (MPC) is a modal indicator designed to decide whether the mode shape used in its computation is a real or complex-valued vector. Its estimate inherits the statistical properties of the corresponding mode shape estimate. While the statistical framework for the uncertainty quantification of modal parameters is well-known and developed in the context of subspace-based system identification methods, uncertainty quantification for the MPC estimate has not been carried out yet. In this paper, the uncertainty quantification of the MPC estimates is developed when the corresponding mode shapes are complex-valued vectors. In this case, the theoretical value of the MPC is strictly lower than 1 and it is shown that the distribution of the MPC estimate can be approximated as Gaussian. The computation of its variance and the resulting confidence intervals of the MPC estimate are developed. The proposed framework is validated in Monte Carlo simulations and illustrated on experimental data of an offshore structure.

Keywords: Uncertainty quantification, Delta method, Ambient excitation, Modal Phase Collinearity, Modal analysis, Mode shape complexity
\end{abstract}

\section{Introduction}

In Operational Modal Analysis, the modal parameters, i.e. natural frequencies, damping ratios and mode shapes, are estimated from vibration measurements, where they are related to the eigenstructure of a linear system [1]. Only the measured output data are required, such as accelerations, displacements, velocities or strains, that are recorded on the structure during unknown, unmeasured, ambient excitation conditions. The resulting modal parameter estimates are never equal to the exact parameters of the structure, since they are computed from data of finite length that is moreover afflicted with measurement noise. They are hence impaired with statistical uncertainties. These uncertainties can be quantified or accounted for, which is often crucial in practice when interpreting the outcome from the related system identification algorithms.

\footnotetext{
(C) $<2021>$. This manuscript version is made available under the CC-BY-NC-ND 4.0 license http://creativecommons. org/licenses/by-nc-nd/4.0/.

${ }^{*}$ Corresponding author; E-mail address: sg@civil.aau.dk
} 
In this context, explicit expressions for the variance computation of the modal parameter estimates have been developed e.g. for subspace-based system identification methods. For example, first-order perturbation theory is used to express the variance of modal parameter estimates in covariance-driven output-only stochastic subspace identification in [2], based on the developments of [3]. An efficient multi-order implementation of the latter scheme for the uncertainty quantification in the stabilization diagram was developed in $[4,5]$, which achieved a significant improvement in the computational complexity compared to the original version of the algorithm. This enabled the application of the uncertainty quantification of the modal parameters in practical applications, e.g. for bridge measurements [6-8]. The framework in [4] was extended to multi-setup subspace identification in [9], and subsequently generalized to the family of input-output and output-only data-driven stochastic subspace identification methods in [10].

For finite element models, the frequencies and mode shapes are usually obtained from the eigenvalue problem concerning the stiffness and mass matrices. System damping is often included by assuming that the damping matrix constitutes a proportional contribution of both aforementioned matrices, and hence can be diagonalized by the eigenvectors of the linear system. The resulting mode shapes are real-valued [11, 12]. While this assumption simplifies the modeling of the mechanical system, it does not apply for OMA, where the estimated eigenstructure always yields complex-valued mode shape vectors [13]. Even when the "true" mode shape of the structure is purely real-valued, its estimate from measurement data is slightly complexvalued due to noise, where the complexity reduces with increasing data length. In other cases, mode shapes can be truly complex-valued vectors due to e.g. non-classical damping [14], gyroscopic effects [15, 16], flutter [17] or closely spaced modes [18]. In practical applications the eigenstructure of the vibrating system can consist of both real and complex-valued mode shapes [19].

The interpretation of mode shapes estimated from the data is facilitated by so-called modal indicators. One of the modal indicators is the Modal Phase Collinearity (MPC) [20], which is a quantity that measures the complexity of a mode shape vector. It is also known under the name Modal Complexity Factor (MCF) $[21,22]$. The MPC indicator is bounded between 0 and 1 , where 1 indicates a real-valued mode shape. The smaller its value, the higher the mode shape complexity. The purpose of this paper is to characterize the statistical distribution of the MPC estimates when the underlying mode shapes are complex-valued vectors. The distribution of the MPC estimate is shown to be asymptotically Gaussian in this case. Subsequently, expressions for its variance computation and the respective confidence intervals are provided. Consequently, it can be assessed whether the underlying mode shape is a complex-valued vector, in particular when the MPC is close but distinct from 1. The confidence intervals for the MPC are indeed a practical metric for OMA, whose pertinence is also shown by their use in commercial software [21].

This paper is organized as follows. Some background on the considered problem is stated in Section 2. The asymptotically Gaussian distribution of the MPC estimate is derived for complex-valued mode shapes in Section 3 based on the delta method [23], from which the variance and confidence intervals follow. An 
application of the proposed framework to measurements from a meteorological mast located in the North Sea is shown in Section 4.

\section{Background on MPC}

In this section, the vibration model is recalled and the statistical parameters of interest are defined. The MPC indicator is introduced for the evaluation of the mode shape complexity, and the problem of uncertainty quantification for this indicator is presented.

\subsection{Mode shape identification}

Assume that the vibration behavior of a viscously damped, linear time-invariant (LTI) structural system with $d$ degrees of freedom is described by the differential equation

$$
M \ddot{q}(t)+C \dot{q}(t)+K q(t)=u(t)
$$

where $t$ denotes continuous time, and matrices $M, C, K \in \mathbb{R}^{d \times d}$ denote mass, damping and stiffness matrices, respectively. Vectors $q(t) \in \mathbb{R}^{d}$ and $u(t) \in \mathbb{R}^{d}$ denote the continuous-time displacements and the unknown external forces, respectively. Let system (1) be observed by sensors measuring e.g. accelerations at $r$ degrees of freedom of the structure. A "true" mode shape $\varphi_{*} \in \mathbb{C}^{r}$ of the structure corresponds to an eigenvector of system (1) at the observed $r$ degrees of freedom. With Operational Modal Analysis (OMA), an estimate $\hat{\varphi}$ of the mode shape can be obtained from the output-only vibration measurements at discrete time instants $t_{k}=k \tau, k=1, \ldots, N$, where $\tau$ is the time step. For the computation of the mode shape estimate the use of methods like stochastic subspace identification (SSI) is assumed, which identify the modal parameters together with their covariance $[2,4,10]$.

\subsection{MPC computation}

In classical mechanical engineering problems, the system matrices from (1) are assumed symmetric and yield a real-valued eigensolution of (1), because $M, C$ and $K$ are diagonalizable by the eigenvectors. In practice, however, the system in (1) can yield complex-valued eigenvectors due to e.g. non-classical formulation of the damping matrix $C$. In addition, the mode shapes estimated from the measured responses are complex-valued, even for simulations of a classically damped system, due to finite data length and noise. In the first case the mode shape is complex-valued due to the physical properties of the system, in the latter case due to estimation errors. Note that a complex-valued mode shape is denoted as complex-valued only if it cannot be turned into a real-valued vector by multiplication with a scalar. In particular, mode shapes with $\Im(\varphi)=a \Re(\varphi)$ with $a \in \mathbb{R}$ correspond to real-valued mode shapes. For the analysis of the complexity of mode shapes, the system is assumed to have distinct eigenvalues. Mode shapes corresponding to double 
modes are linear combinations of some basis vectors and can thus be arbitrarily complex, so complexity indicators like the MPC cannot be directly applied in this case.

A geometric depiction of the components of a complex mode shape is illustrated in Figure 1. It can be seen that the mode shape components plotted in the complex plain align around one axis and exhibit small imaginary parts for a mode shape with low degree of complexity (left) and show significant dispersion in the complex plain for a mode shape with high complexity (right).
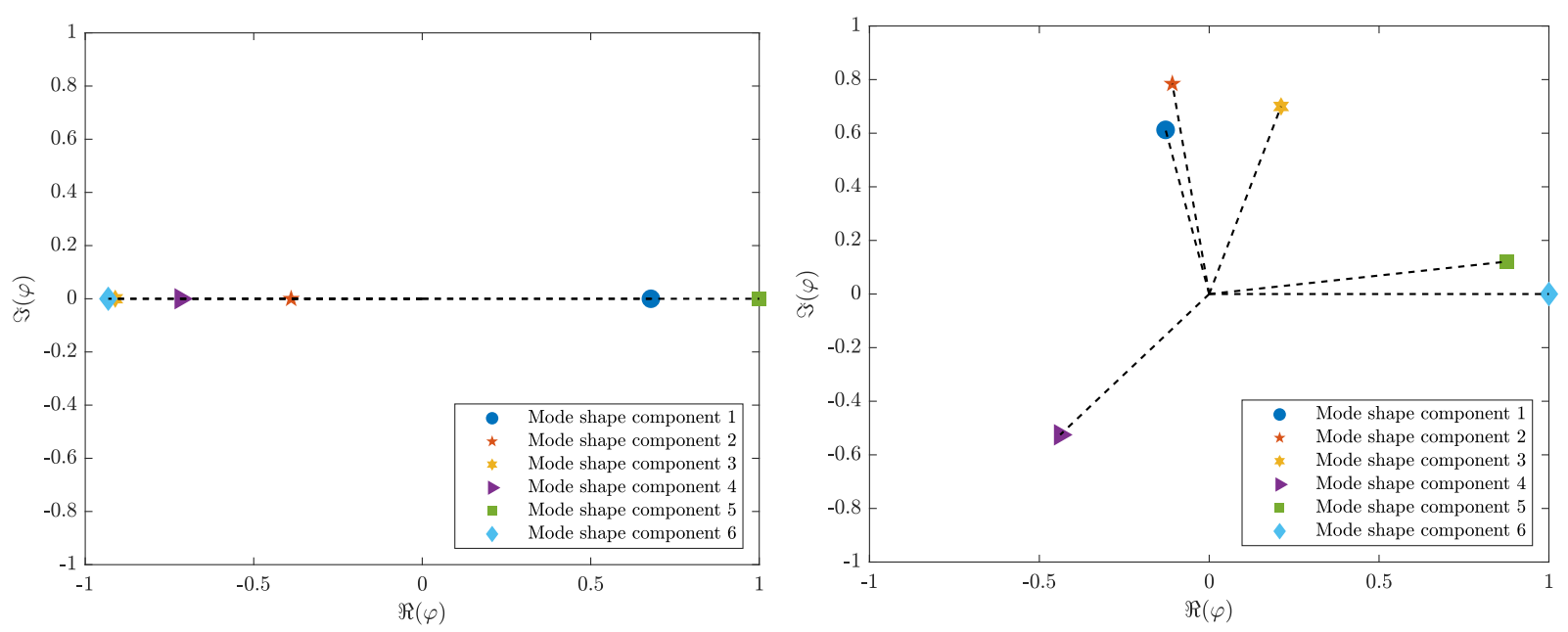

Figure 1: Components of mode shape vector with low complexity (left) and high complexity (right).

The complexity of the mode shape can be quantified by the Modal Phase Collinearity (MPC), which is also called Modal Complexity Factor (MCF), e.g. in [21, 22]. There are different definitions of this indicator in the literature that are equivalent [24], and which are recalled here for the sake of completeness. Define the scalar products $S_{x x}=\Re(\varphi)^{T} \Re(\varphi), S_{y y}=\Im(\varphi)^{T} \Im(\varphi)$ and $S_{x y}=S_{y x}=\Re(\varphi)^{T} \Im(\varphi)$, and the matrix

$$
S=\left[\begin{array}{ll}
S_{x x} & S_{x y} \\
S_{y x} & S_{y y}
\end{array}\right] \in \mathbb{R}^{2 \times 2} .
$$

Then, the definition of $\operatorname{MPC}(\varphi)$ from $[20,25]$ states

$$
\operatorname{MPC}(\varphi) \triangleq \frac{\left(\lambda_{1}^{S}-\lambda_{2}^{S}\right)^{2}}{\left(\lambda_{1}^{S}+\lambda_{2}^{S}\right)^{2}},
$$

where $\lambda_{1}^{S}$ and $\lambda_{2}^{S}$ are the eigenvalues of matrix $S$. From this expression it follows

$$
\operatorname{MPC}(\varphi)=\frac{\left(S_{x x}-S_{y y}\right)^{2}+4 S_{x y}^{2}}{\left(S_{x x}+S_{y y}\right)^{2}} .
$$

Furthermore, based on the definition of the Modal Assurance Criterion (MAC) [26]

$$
\operatorname{MAC}(\varphi, \psi) \triangleq \frac{\left|\varphi^{H} \psi\right|^{2}}{\varphi^{H} \varphi \psi^{H} \psi}=\frac{\varphi^{H} \psi \psi^{H} \varphi}{\varphi^{H} \varphi \psi^{H} \psi},
$$


the MAC value between the mode shape and its complex conjugate is equivalent to the MPC, yielding $\operatorname{MPC}(\varphi)=\operatorname{MAC}(\varphi, \bar{\varphi})$. The equivalences are shown in detail in Appendix A. In the remainder of this paper, expression (4) will be used.

Consequently, similar to the MAC, the MPC indicator is bounded between 0 and 1 . For real mode shapes its value is 1 , and the lower the MPC the more complex the mode shape. Since the MPC is a function of the mode shape, the statistical properties of the mode shape estimate propagate to the MPC estimate, based on which its asymptotic distribution function can be inferred. The distribution of the MPC estimates is referred to as $M P C$ distribution in the following.

\section{3. $M P C$ distribution}

Independent realizations of the MPC estimate yield a histogram showing the MPC distribution. Different MPC distributions that may appear in practice are illustrated in the following example.

Consider a 6 DOF chain-like system as illustrated in Figure 2 that, for any consistent set of units, is modeled with spring stiffnesses $k_{1}=k_{3}=k_{5}=100$ and $k_{2}=k_{4}=k_{6}=200$, mass $m_{i}=1 / 20$ and a damping matrix $C$ yielding both complex and real mode shapes, defined by

$$
C=\left[\begin{array}{cccccc}
0.2007 & -0.0236 & 0.0595 & 0.1170 & 0.0736 & 0.1341 \\
-0.0236 & 0.0192 & -0.1054 & -0.0748 & -0.1617 & -0.0773 \\
0.0595 & -0.1054 & 0.1263 & -0.0297 & -0.0843 & 0.0286 \\
0.1170 & -0.0747 & -0.0297 & 0.2570 & -0.0748 & 0.1227 \\
0.0735 & -0.1617 & -0.0842 & -0.0748 & -0.0601 & -0.1141 \\
0.1341 & -0.0773 & 0.0286 & 0.1226 & -0.1142 & 0.2558
\end{array}\right] .
$$

This damping matrix is constructed such that theoretical mode shape 5 is slightly complex $\left(\mathrm{MPC}_{5}=0.9787\right)$, mode shape 1 is close to real $\left(\mathrm{MPC}_{1}=0.9994\right)$, and mode shape 3 is purely real $\left(\mathrm{MPC}_{3}=1\right)$. While no particular physical interpretation for the components of the damping matrix is considered, the mechanical system is stable and exhibits mode shapes of different complexity, which are used to illustrate different representative cases of MPC distributions in this paper. The exact modal parameters of the system are depicted in Table 1.

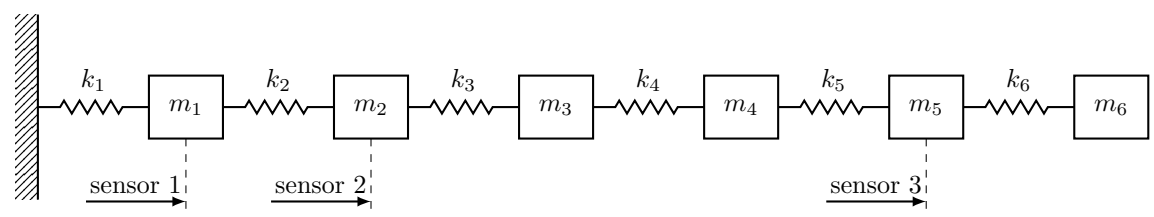

Figure 2: Illustration of 6 DOF chain system used for Monte Carlo simulation. 
Table 1: Exact modal parameters of the chain system.

\begin{tabular}{l|rrrrrr}
\hline Mode & 1 & 2 & 3 & 4 & 5 & 6 \\
\hline Natural frequency $f_{i}(\mathrm{~Hz})$ & 1.936 & 5.618 & 8.682 & 14.494 & 15.798 & 17.007 \\
Damping ratio $\zeta_{i}(\%)$ & 2 & 2.005 & 2 & 2 & 2 & 2.005 \\
Modal phase collinearity $\mathrm{MPC}_{i}$ & 0.9994 & $\approx 1$ & 1 & 1 & 0.9787 & 0.9799 \\
\hline
\end{tabular}

The system is excited by a random white noise signal in all DOFs and sampled with a frequency of $50 \mathrm{~Hz}$ for 2000 seconds. The responses are measured at DOFs 1, 2 and 5. Gaussian white noise with 5\% of the standard deviation of the output is added to the response at each channel. The computations are performed in a Monte Carlo setup with $m=1000$ realizations of the described signal. For the estimation of the mode shapes, the output-only data-driven subspace-based system identification with the unweighted principal component (SSI-UPC) [27] is used, where the estimates are obtained at system order 12, using 15 time lags for the computation of the data Hankel matrix. The six modes of the system are tracked in each simulation. Figure 3 shows the histograms of the estimates of $\mathrm{MPC}_{5}, \mathrm{MPC}_{1}, \mathrm{MPC}_{3}$ that are computed from the corresponding mode shape estimate of modes 5, 1 and 3, respectively.
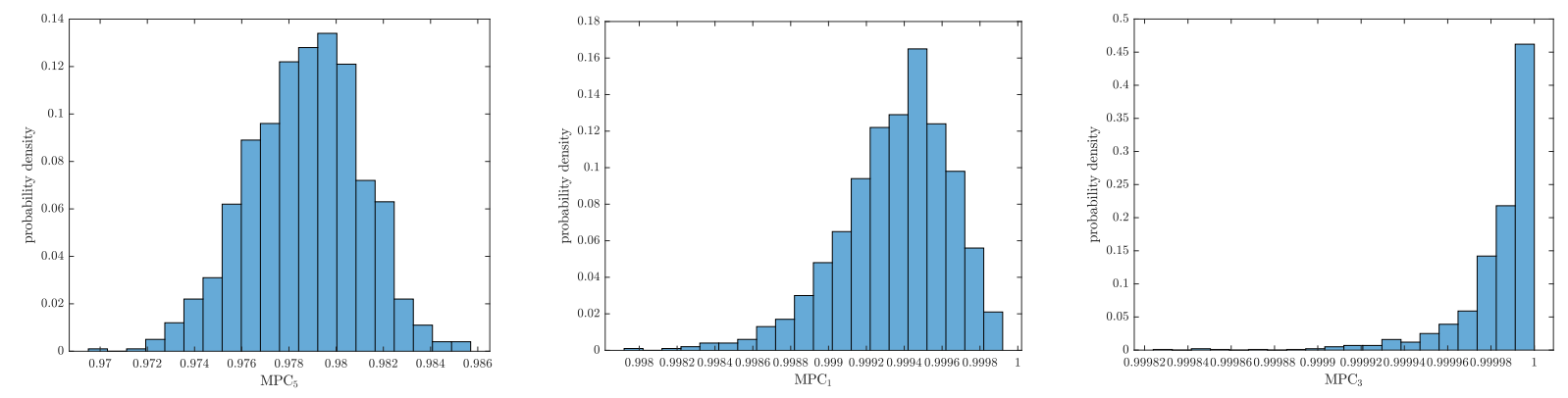

Figure 3: Histograms of MPC from simulations of non-proportionally damped chain-like system with $N=100,000$ samples. $\mathrm{MPC}_{5}$ left, $\mathrm{MPC}_{1}$ middle, $\mathrm{MPC}_{3}$ right.

These histograms illustrate three distinctively different cases of MPC distributions. Indeed, it can be observed that these distributions depend heavily on the complexity of the considered mode shape. It seems that the distribution of $\mathrm{MPC}_{5}$ in Figure 3 (left) is Gaussian, corresponding to the complex mode shape, and the distribution of $\mathrm{MPC}_{1}$ in Figure 3 (middle) corresponding to the close-to-real mode shape looks more skew. However, the distribution of $\mathrm{MPC}_{3}$ in Figure 3 (right) corresponding to the real mode shape is very different. In the following section, the distributions of $\mathrm{MPC}_{5}$ and $\mathrm{MPC}_{1}$ corresponding to complex mode shapes are characterized in detail in order to quantify the uncertainty of single MPC estimates from measurement data in appropriate confidence intervals. The case of $\mathrm{MPC}_{3}$, which corresponds to a purely real-valued mode shape, is not treated in this work. 


\section{Gaussian approximation for the distribution of MPC}

In this section a Gaussian approximation is developed for the MPC estimate when the underlying theoretical mode shape $\varphi_{*}$ is complex, i.e. the theoretical value $\operatorname{MPC}\left(\varphi_{*}\right)$ is smaller than 1 . Since the MPC estimate is a function of the mode shape estimate, the statistical properties of the mode shape estimate propagate to the MPC. We assume that the mode shapes originate from a system identification method that provides Gaussian estimates together with an estimate of the covariance, e.g. subspace identification $[2,4,10]$. A tool to propagate the covariance of a Gaussian variable is the delta method, which is applied to the expression for the MPC in the next section.

\subsection{Gaussian approximation of MPC distribution with the first-order delta method}

The delta method is a statistical tool that helps to estimate the covariance of a function of a Gaussian variable, from where confidence intervals can be derived. It has been applied successfully in engineering problems over the last decade, in particular for the uncertainty quantification of modal parameters $[7,9,10]$ or vibration-based damage diagnosis [28-30].

With the first-order delta method, the probability distribution of a function of an asymptotically Gaussian vector can be characterized as also asymptotically Gaussian. The MPC is a function of the mode shape, which is assumed to be estimated from data with methods like stochastic subspace identification (SSI) [27]. For these methods, it has been shown that mode shape estimate $\hat{\varphi}$ converges to the true mode shape $\varphi_{*}$ when the data length $N$ increases, and that the estimates are indeed asymptotically Gaussian distributed $[10,31]$. This means that the probability distribution of the real and imaginary parts of the mode shape estimate can be well-approximated by a Gaussian distribution when $N$ is large enough. In mathematical terms, this property is expressed by the Central Limit Theorem (CLT)

$$
\sqrt{N}\left(\left[\begin{array}{l}
\Re(\hat{\varphi}) \\
\Im(\hat{\varphi})
\end{array}\right]-\left[\begin{array}{l}
\Re\left(\varphi_{*}\right) \\
\Im\left(\varphi_{*}\right)
\end{array}\right]\right) \stackrel{\mathcal{L}}{\rightarrow} \mathcal{N}\left(0, \Sigma_{\varphi_{*}}\right),
$$

where $\Re(\cdot)$ and $\Im(\cdot)$ are the real and imaginary parts of a complex variable, " $\mathcal{L}$ " denotes convergence in distribution, $\mathcal{N}(\mu, \Sigma)$ is a Gaussian distributed variable with mean $\mu$ and covariance $\Sigma$, and $\Sigma_{\varphi_{*}} \in \mathbb{R}^{2 r \times 2 r}$ is called the asymptotic mode shape covariance. The computation of a consistent estimate $\widehat{\Sigma}_{\varphi_{*}}$ of the asymptotic mode shape covariance is considered for different SSI methods e.g. in $[2,4,10]$. Remark that thanks to property (6), the distribution of the mode shape estimate can be approximated by

$$
\left[\begin{array}{l}
\Re(\hat{\varphi}) \\
\Im(\hat{\varphi})
\end{array}\right] \approx \mathcal{N}\left(\left[\begin{array}{l}
\Re\left(\varphi_{*}\right) \\
\Im\left(\varphi_{*}\right)
\end{array}\right], \frac{1}{N} \widehat{\Sigma}_{\varphi_{*}}\right) .
$$

Now consider the function $\operatorname{MPC}(\varphi)$ that computes the MPC indicator in (4). In order to apply the delta method, its derivative $\mathcal{J}_{\varphi}^{\mathrm{MPC}}$ with respect to the real and imaginary parts of the mode shape is required, 
and it needs to be ensured that this derivative is non-zero at its limit value [23]. It writes

$$
\mathcal{J}_{\varphi}^{\mathrm{MPC}}=\left[\begin{array}{ll}
\frac{\partial \mathrm{MPC}}{\partial \Re(\varphi)} & \frac{\partial \mathrm{MPC}}{\partial \Im(\varphi)}
\end{array}\right] \in \mathbb{R}^{1 \times 2 r},
$$

where, based on the definition of the MPC in (4), it follows

$$
\begin{aligned}
\frac{\partial \mathrm{MPC}}{\partial \Re(\varphi)} & =\frac{\frac{\partial\left(\left(S_{x x}-S_{y y}\right)^{2}+4 S_{x y}^{2}\right)}{\partial \Re(\varphi)} c^{2}-\left(\left(S_{x x}-S_{y y}\right)^{2}+4 S_{x y}^{2}\right) \frac{\partial c^{2}}{\partial \Re(\varphi)}}{c^{4}} \\
& =\frac{4\left(S_{x x}-S_{y y}\right) \Re(\varphi)^{T}+8 S_{x y} \Im(\varphi)^{T}}{c^{2}}-\frac{4 \operatorname{MPC}(\varphi) \Re(\varphi)^{T}}{c}, \\
\frac{\partial \mathrm{MPC}}{\partial \Im(\varphi)} & =\frac{\frac{\partial\left(\left(S_{x x}-S_{y y}\right)^{2}+4 S_{x y}^{2}\right)}{\partial \Im(\varphi)} c^{2}-\left(\left(S_{x x}-S_{y y}\right)+4 S_{x y}^{2}\right) \frac{\partial c^{2}}{\partial \Im(\varphi)}}{c^{4}} \\
& =\frac{4\left(S_{y y}-S_{x x}\right) \Im(\varphi)^{T}+8 S_{x y} \Re(\varphi)^{T}}{c^{2}}-\frac{4 \operatorname{MPC}(\varphi) \Im(\varphi)^{T}}{c},
\end{aligned}
$$

where $c=S_{x x}+S_{y y}$. An estimate $\mathcal{J}_{\hat{\varphi}}^{\mathrm{MPC}}$ of the derivative $\mathcal{J}_{\varphi_{*}}^{\mathrm{MPC}}$ is easily obtained by evaluating (9)-(10) at the mode shape estimate $\hat{\varphi}$. It can be shown that $\mathcal{J}_{\varphi_{*}}^{\mathrm{MPC}}$ is non-zero if and only if the underlying theoretical MPC value is different from 0 and 1 , as detailed in Appendix B.1, i.e.

$$
\forall \varphi_{*} \in \mathbb{C}^{r}: \quad \operatorname{MPC}\left(\varphi_{*}\right) \notin\{0,1\} \Longleftrightarrow \mathcal{J}_{\varphi_{*}}^{\mathrm{MPC}} \neq 0 .
$$

Hence, if a mode shape $\varphi_{*}$ is estimated with $\operatorname{MPC}\left(\varphi_{*}\right) \notin\{0,1\}$, which in particular is the case for estimates of complex mode shapes, then the derivative $\mathcal{J}_{\varphi_{*}}^{\mathrm{MPC}}$ is non-zero. Subsequently, a first-order Taylor expansion writes

$$
\operatorname{MPC}(\hat{\varphi})=\operatorname{MPC}\left(\varphi_{*}\right)+\mathcal{J}_{\varphi_{*}}^{\operatorname{MPC}}\left(\left[\begin{array}{l}
\Re(\hat{\varphi}) \\
\Im(\hat{\varphi})
\end{array}\right]-\left[\begin{array}{l}
\Re\left(\varphi_{*}\right) \\
\Im\left(\varphi_{*}\right)
\end{array}\right]\right)+o\left(\left\|\hat{\varphi}-\varphi_{*}\right\|\right),
$$

and the distribution of the MPC estimate can be approximated as Gaussian by means of the delta method [23], following from (6) and (12) as

$$
\sqrt{N}\left(\operatorname{MPC}(\hat{\varphi})-\operatorname{MPC}\left(\varphi_{*}\right)\right) \stackrel{\mathcal{L}}{\rightarrow} \mathcal{N}\left(0, \sigma_{\mathrm{MPC}}^{2}\right),
$$

where $\sigma_{\mathrm{MPC}}^{2} \triangleq \mathcal{J}_{\varphi_{*}}^{\mathrm{MPC}} \Sigma_{\varphi_{*}}\left(\mathcal{J}_{\varphi_{*}}^{\mathrm{MPC}}\right)^{T}$. Note that this property only holds if $\sigma_{\mathrm{MPC}}^{2} \neq 0$, which is shown in Appendix B.2. Equation (13) shows two important results. First, it states that the distribution of the MPC estimate is Gaussian when $\operatorname{MPC}\left(\varphi_{*}\right) \notin\{0,1\}$, i.e. in particular when the mode shape is complex-valued. Second, it provides a practical formula for the computation of the MPC variance. An estimate of $\sigma_{\mathrm{MPC}}^{2}$ is obtained as $\hat{\sigma}_{\mathrm{MPC}}^{2}=\mathcal{J}_{\hat{\varphi}}^{\mathrm{MPC}} \widehat{\Sigma}_{\varphi_{*}}\left(\mathcal{J}_{\hat{\varphi}}^{\mathrm{MPC}}\right)^{T}$, and the variance of $\operatorname{MPC}(\hat{\varphi})$ is then easily computed based on (13) as

$$
\operatorname{var}(\operatorname{MPC}(\hat{\varphi})) \approx \frac{1}{N} \hat{\sigma}_{\mathrm{MPC}}^{2}=\frac{1}{N} \mathcal{J}_{\hat{\varphi}}^{\mathrm{MPC}} \widehat{\Sigma}_{\varphi_{*}}\left(\mathcal{J}_{\hat{\varphi}}^{\mathrm{MPC}}\right)^{T} .
$$

Hence, the distribution of the MPC estimate can be approximated by

$$
\operatorname{MPC}(\hat{\varphi}) \approx \mathcal{N}\left(\operatorname{MPC}\left(\varphi_{*}\right), \frac{1}{N} \hat{\sigma}_{\mathrm{MPC}}^{2}\right)
$$


Since the distribution is approximately Gaussian, confidence intervals can be easily determined for a given confidence level. For example, the $95 \%$ interval is given by

$$
\left(\operatorname{MPC}(\hat{\varphi})-2 \cdot \frac{1}{\sqrt{N}} \hat{\sigma}_{\mathrm{MPC}}, \operatorname{MPC}(\hat{\varphi})+2 \cdot \frac{1}{\sqrt{N}} \hat{\sigma}_{\mathrm{MPC}}\right) .
$$

The procedure for the Gaussian approximation of the MPC estimate is summarized in Algorithm 1.

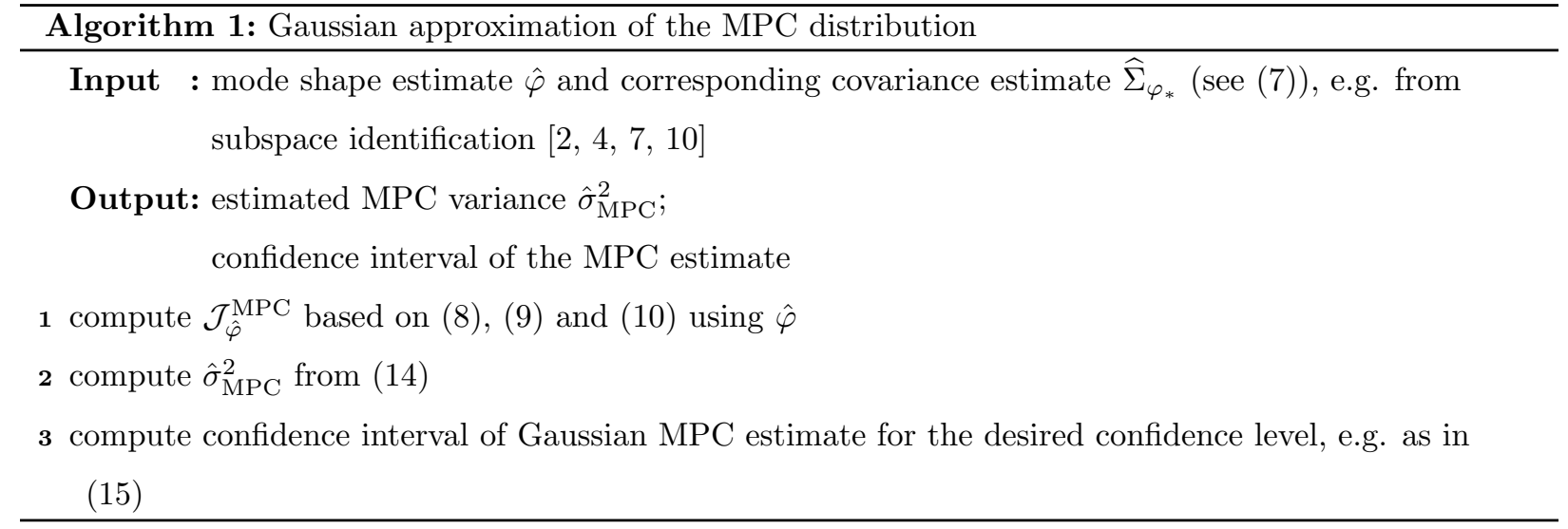

\subsection{Assessment of the MPC distribution in Monte Carlo simulations}

In this section the Gaussian approximation of the MPC distribution is validated in Monte Carlo simulations for $\mathrm{MPC}_{5}$ and $\mathrm{MPC}_{1}$ that are computed on complex mode shapes, see also Figure 3.

For each Monte Carlo simulation $j=1, \ldots, m$ with $m=1000$ consider the estimated MPC value $\mathrm{MPC}_{\mathrm{MC}, j}$ and denote $\mathrm{MPC}_{\mathrm{MC}} \in \mathbb{R}^{m \times 1}$ the vector of all the MPC estimates from $m$ Monte Carlo simulations. Then it is straightforward to compute their sample mean $\overline{\mathrm{MPC}}_{\mathrm{MC}}$ and their sample standard deviation $s_{\mathrm{MC}}=\sqrt{\operatorname{var}\left(\mathrm{MPC}_{\mathrm{MC}}\right)}$. The sample standard deviation $s_{\mathrm{MC}}$ is an estimate of the standard deviation of the MPC distribution evaluated for a fixed data length $N$, computed on 1000 data sets. The standard deviation computed with the delta method in this paper is based on the Gaussian approximation, and requires only one data set for its computation. Its validation is carried out as follows. The variance computation based on the delta method (14) is considered for each of the Monte Carlo simulations, and denote $\sigma_{\mathrm{DM}, j}$ as the standard deviation of the MPC computed from the $j$-th data set. Their mean $\bar{\sigma}_{\mathrm{DM}}$ should match the sample standard deviation $s_{\mathrm{MC}}$, since both are estimates of the MPC standard deviation from the same number of data sets. This is analyzed in the histograms of $\sigma_{\mathrm{DM}, j}$ along with $s_{\mathrm{MC}}$ for $\mathrm{MPC}_{5}$ and $\mathrm{MPC}_{1}$, shown in Figure 4 and summarized in Table 2. It can be observed that the histograms of the standard deviations computed by the delta method are centered around the sample standard deviation of the Monte Carlo simulations, and $s_{\mathrm{MC}}$ and $\bar{\sigma}_{\mathrm{DM}}$ are indeed close. Since $s_{\mathrm{MC}}$ is computed from a finite number of Monte Carlo simulations, it has some uncertainty that can be easily evaluated [32] considering the MPC distribution is approximately 
Gaussian for $N$ large enough. The respective $95 \%$ confidence intervals of $s_{\mathrm{MC}}$ are $[2.753,3.006]$ for $\mathrm{MPC}_{1}$ and $[22.740,24.826]$ for $\mathrm{MPC}_{5}$, containing $\bar{\sigma}_{\mathrm{DM}}$ in both cases. This validates the correct computation of the MPC standard deviation with the proposed method on average. Furthermore, the computed standard deviations $\sigma_{\mathrm{DM}, j}$ exhibit small deviations among the different data sets. Their spread $s_{\sigma_{\mathrm{DM}}}$ can be evaluated from the sample standard deviation of the computed $\sigma_{\mathrm{DM}, j}$ 's and is shown in Table 2. Its values are around $9 \%$ for $\mathrm{MPC}_{5}$ and $25 \%$ for $\mathrm{MPC}_{1}$. This suggests that on average any variance estimate can be used to approximate the histogram of the respective MPC.
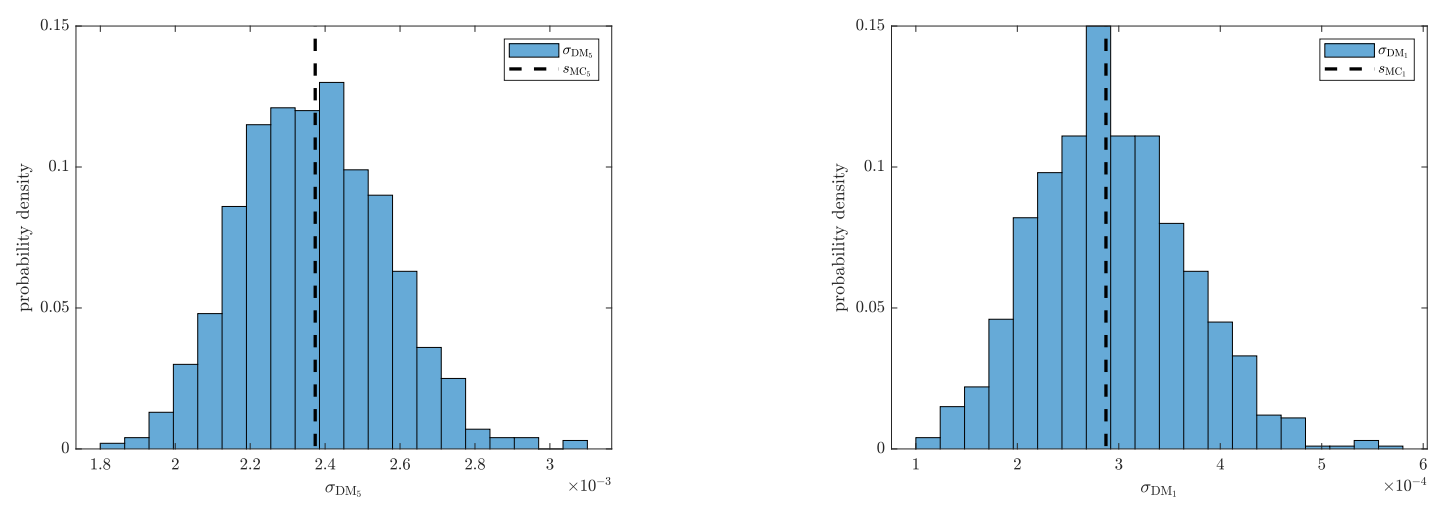

Figure 4: Histograms of delta method-based standard deviations of $\mathrm{MPC}_{5}$ (left) and $\mathrm{MPC}_{1}$ (right).

Table 2: Comparison of the Monte Carlo and delta method-based statistical properties of $\mathrm{MPC}_{1}$ and $\mathrm{MPC}_{5}$.

\begin{tabular}{ccccc}
\hline Data length & $\mathrm{MPC}$ & $s_{\mathrm{MC}} \times 10^{-4}$ & $\bar{\sigma}_{\mathrm{DM}} \times 10^{-4}$ & $s_{\sigma_{\mathrm{DM}}} \times 10^{-4}$ \\
\hline \multirow{2}{*}{$N=100,000$} & $\mathrm{MPC}_{1}$ & 2.874 & 2.926 & 0.739 \\
& $\mathrm{MPC}_{5}$ & 23.737 & 23.697 & 1.939 \\
\hline
\end{tabular}

Finally, the delta method-based variance obtained from one data set is used to compute the Gaussian distribution function of the respective MPC. The corresponding distributions are shown in Figure 5. It appears that the proposed variance computation scheme is good for $\mathrm{MPC}_{5}$ (left) and adequate for $\mathrm{MPC}_{1}$ (right). Based on that it can be conjectured that the density approximation computed with the delta method can be used to encompass the empirical distributions of the $\mathrm{MPC}_{5}$ and $\mathrm{MPC}_{1}$ well.

\subsection{Influence of data length on the MPC distribution}

As illustrated by Figure 5 (right), the histogram of $\mathrm{MPC}_{1}$ estimates cannot fully satisfy a Gaussian assumption due to its proximity to the boundary at 1 . Since the theoretical value of $\mathrm{MPC}_{1}$ is 0.9994 , and hence distinct from 1, Property (13) justifies a Gaussian distribution for the estimates of $\mathrm{MPC}_{1}$, provided 

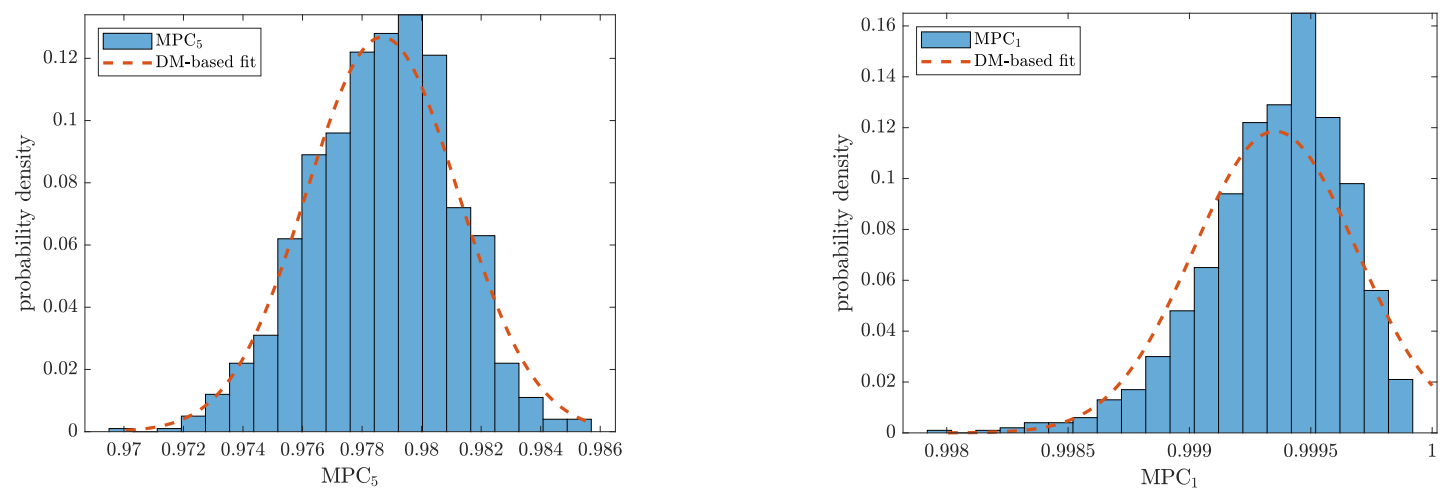

Figure 5: Gaussian fits to empirical probability distribution of $\mathrm{MPC}_{5}$ (left) and $\mathrm{MPC}_{1}$ (right).

the data length $N$ is large enough. This convergence property will be illustrated now with different data lengths.

Three histograms of $\mathrm{MPC}_{1}$ estimates together with the delta method-based PDF fits are shown in Figure 6 for different data lengths. It can be observed that the density approximations get closer to the histogram as the data length increases. This suggests that the $\mathrm{MPC}_{1}$ estimate is indeed Gaussian, and that the first-order delta method can accurately approximate the distribution when a sufficient amount of data is available. However, when not enough data are available the distribution fits do not lie entirely in the unit interval $[0,1]$. In particular, the resulting confidence intervals would contain the value 1 and be in part outside the unit interval, which is a contradiction to the assumption of the mode being complex and makes a Gaussian approximation indeed questionable in this case. Thus, complex mode shapes with MPCs close to 1 require a sufficient amount of data for an appropriate Gaussian approximation. Since the variance of the distribution is decreasing with $N$, the confidence intervals are likely to become small enough to be within the unit interval for large $N$.

The corresponding sample means and standard deviations of the Monte Carlo simulations, together with the mean values of the standard deviations computed with the delta method $\left(\bar{\sigma}_{\mathrm{DM}}\right)$ and their spreads $\left(s_{\sigma_{\mathrm{DM}}}\right)$ are depicted in Table 3. As $N$ increases, the mean of the Monte Carlo histogram stabilizes around the exact value of $\mathrm{MPC}_{1}$, as expected. The mean of the standard deviations computed with the delta method for each of the Monte Carlo simulations and the Monte Carlo sample standard deviation are close, and nearly identical for higher $N$. The standard deviation decreases, and its spread also decreases from $57 \%$ at $N=$ 10,000 to $9 \%$ at $N=1,000,000$.

These examples show that for almost real and only slightly complex mode shapes, for which the MPC is close to the boundary, the Gaussian assumption may require long data sets to be realistically satisfied. For example, $\mathrm{MPC}_{5}$ has a satisfying approximation for $N=100,000$ whereas $\mathrm{MPC}_{1}$ requires 10 times the amount of data for a similar fit. Moreover, when the mode is purely real and the MPC is at the boundary 

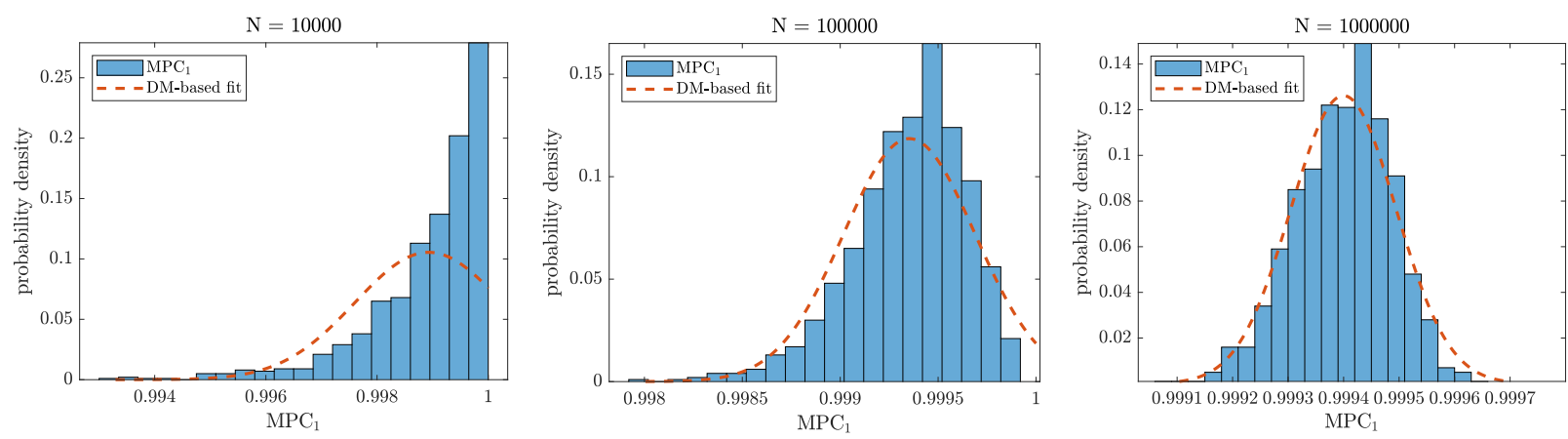

Figure 6: Histograms of $\mathrm{MPC}_{1}$ estimates with different data lengths

Table 3: Comparison of the Monte Carlo and delta method-based statistical properties of MPC .

\begin{tabular}{cccccc}
\hline Data length & $\mathrm{MPC}_{1}$ & $\overline{\mathrm{MPC}}_{\mathrm{MC}}$ & $s_{\mathrm{MC}} \times 10^{-4}$ & $\bar{\sigma}_{\mathrm{DM}} \times 10^{-4}$ & $s_{\sigma_{\mathrm{DM}}} \times 10^{-4}$ \\
\hline$N=10,000$ & 0.9994 & 0.9989 & 10.58 & 14.760 & 8.443 \\
$N=100,000$ & 0.9994 & 0.9994 & 2.874 & 2.926 & 0.739 \\
$N=1,000,000$ & 0.9994 & 0.9994 & 0.872 & 0.881 & 0.083 \\
\hline
\end{tabular}

(such as $\mathrm{MPC}_{3}$ ), the Gaussian assumption is not feasible anymore as shown by (13). Such cases require the proposed approach to be replaced by a better suited framework, whose development is beyond the scope of this paper.

\section{Application}

This section presents a practical application of the uncertainty quantification framework from Section 3. The tested structure is a full scale meteorological mast, located in the west of the Dogger Bank site in the North Sea, supported by a novel concept of offshore foundation at that time, namely a Mono Bucket foundation. The foundation and the meteorological mast are shown in Figure 7.

The vibration response of the structure to ambient excitation (wind, waves and current) is continuously recorded by 8 accelerometers at a sampling frequency of $20 \mathrm{~Hz}$, and the measurements are decimated to $6.67 \mathrm{~Hz}$. A data set of length $N=400,000$ is selected to estimate the modal parameters and subsequently to perform the statistical analysis of the MPC estimates. The modal parameters are estimated at model orders ranging from $n_{\min }=10$ to $n_{\max }=40$ using the data-driven SSI-UPC algorithm. The covariances of the modal parameters are computed using the numerically efficient implementation developed in [4] in combination with [10]. The variances of the MPC estimates are computed with (14) from Section 3, as detailed in Algorithm 1.

One of the engineering tools in OMA is a stabilization diagram, which is used to identify the physical 

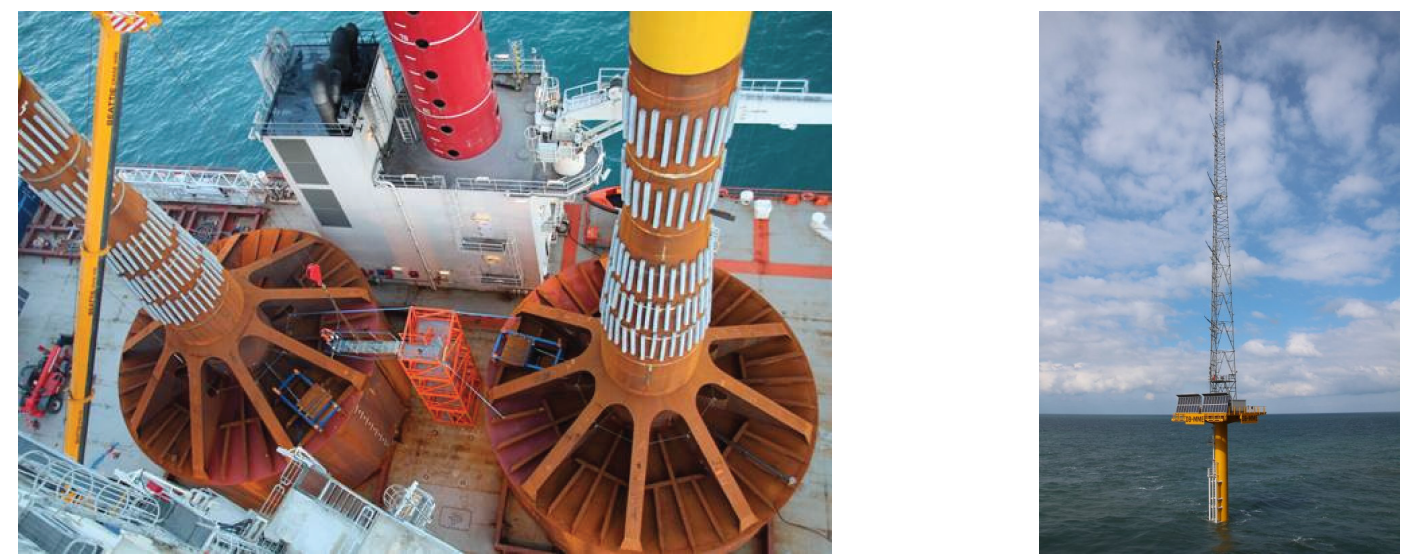

Figure 7: Twin Mono Bucket foundations on board of the installation vessel (left). Meteorological mast at Dogger Bank (right). Both photos available by the courtesy of Universal Foundation A/S.

structural modes, considering the order of the system is unknown. The system order determines the number of identified modal parameters. Different system orders result in different estimates of the modal parameters that may vary at different orders. Some of those parameters correspond to non-physical, noise and mathematical poles. Conversely to the physical poles, which are stable in the stabilization diagram, the spurious estimates have high dispersion. One of the applications of the uncertainty quantification of modal parameters is to filter the stabilization diagrams from modes that exhibit high variances, as showed e.g. in $[4,7]$. In the following, a threshold on the coefficient of variation of the natural frequency estimates is set to 0.001 to filter the uncertain modes, see Figure 8 .

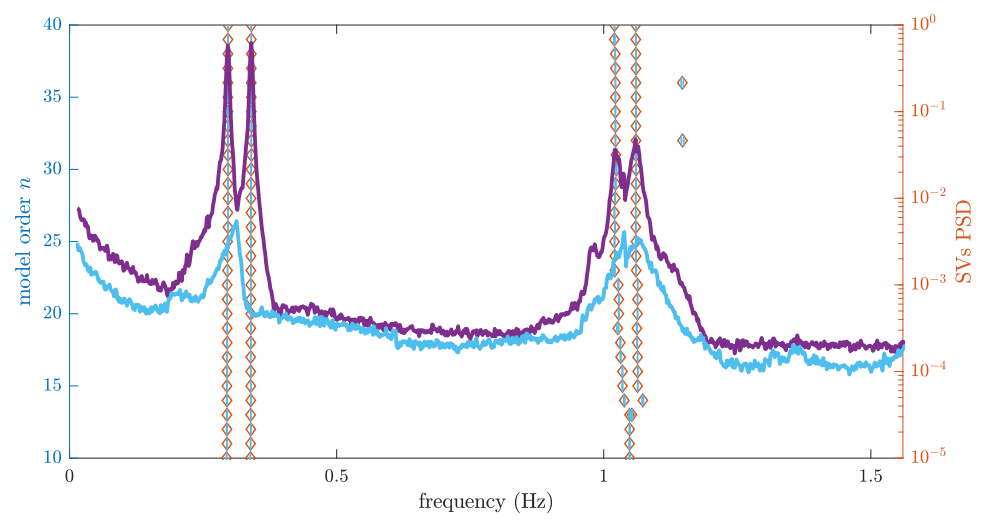

Figure 8: Stabilization diagram of natural frequencies for maximum model order 40.

Four fundamental frequencies of the mast are then easily identified, as detailed in Table 4. Modes 1 and 2, as well as modes 3 and 4 have close but distinct frequencies. Indeed, the meteorological mast is not axis-symmetric [33] and its modes are not double modes. Subsequently, the MPCs computed from the corresponding mode shape estimates are analyzed. An illustration of the real and imaginary components 
Table 4: Modal parameters of the mast estimated at model order 40.

\begin{tabular}{l|rrrr}
\hline Mode & 1 & 2 & 3 & 4 \\
\hline Natural frequency $f_{i}(\mathrm{~Hz})$ & 0.296 & 0.340 & 1.020 & 1.060 \\
Damping ratio $\zeta_{i}(\%)$ & 1.044 & 0.562 & 1.534 & 1.010 \\
Modal phase collinearity $\mathrm{MPC}_{i}$ & 0.9992 & 0.9992 & 0.8466 & 0.8234 \\
\hline
\end{tabular}
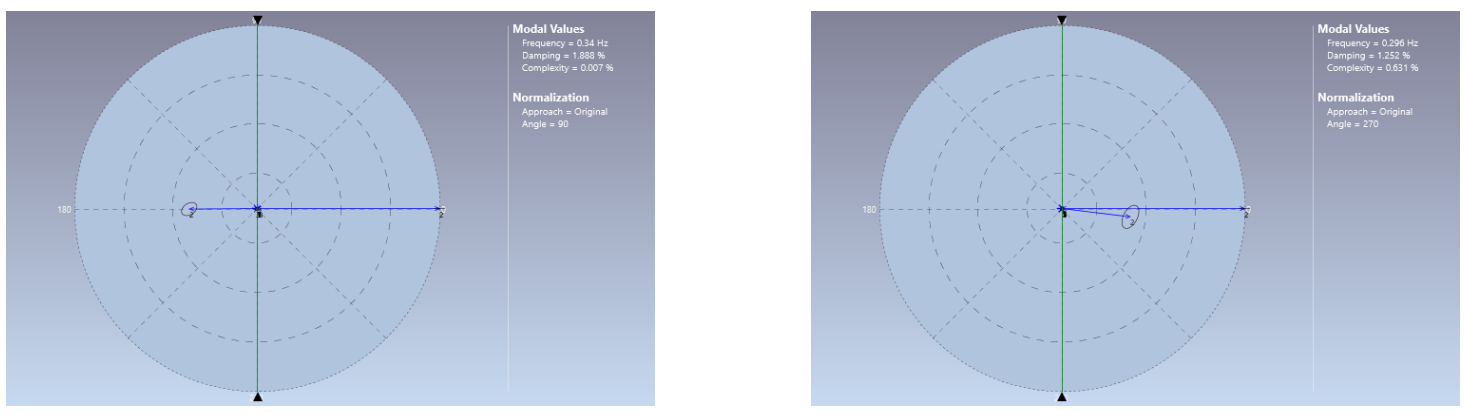

Figure 9: Plots of real and imaginary components of the first (left) and the second (right) mode shape with their corresponding confidence ellipsoids. Both plots established in ARTEMIS ModAL Pro 6.0.

of the first two mode shapes, together with their confidence intervals, is presented in Figure 9 . It can be observed that the estimated components are aligned around one direction and their dispersion is very low. This indicates that their complexity is also low and consequently the underlying MPC estimates are in the vicinity of 1 for the first two modes. As shown in Table 4, the MPCs for all mode shapes are strictly smaller than 1 , but some are so close to 1 that a confidence interval is needed to decide about the complexity of the mode shape.

The evaluation of the mode shape complexity is carried out by using the confidence intervals computed based on the MPC variance estimates from Algorithm 1. In Figure 10 the 95\% confidence intervals are shown for the modal alignments of the first four modes.

First, it can be observed that the MPC estimates of the first two mode shapes are close to 1 . For the first mode shape, whose components are aligned around one axis in Figure 9 (left), the confidence interval of the MPC estimates severely surpass the theoretical boarder of the MPC domain at 1 for nearly all model orders. This suggests that the assumption of a complex-valued mode shape that has been made in the present uncertainty quantification framework is not satisfied, and thus the first mode shape may be realvalued. Note that the MPC distribution for real-valued mode shapes cannot be approximated by a Gaussian function, and its analysis is outside the scope of this paper. The confidence bounds for the MPC estimates corresponding to the next three modes are (nearly all) well separated from the boarder at 1, indicating that a Gaussian distribution is a possible fit for them, since for each case, the confidence interval around the 

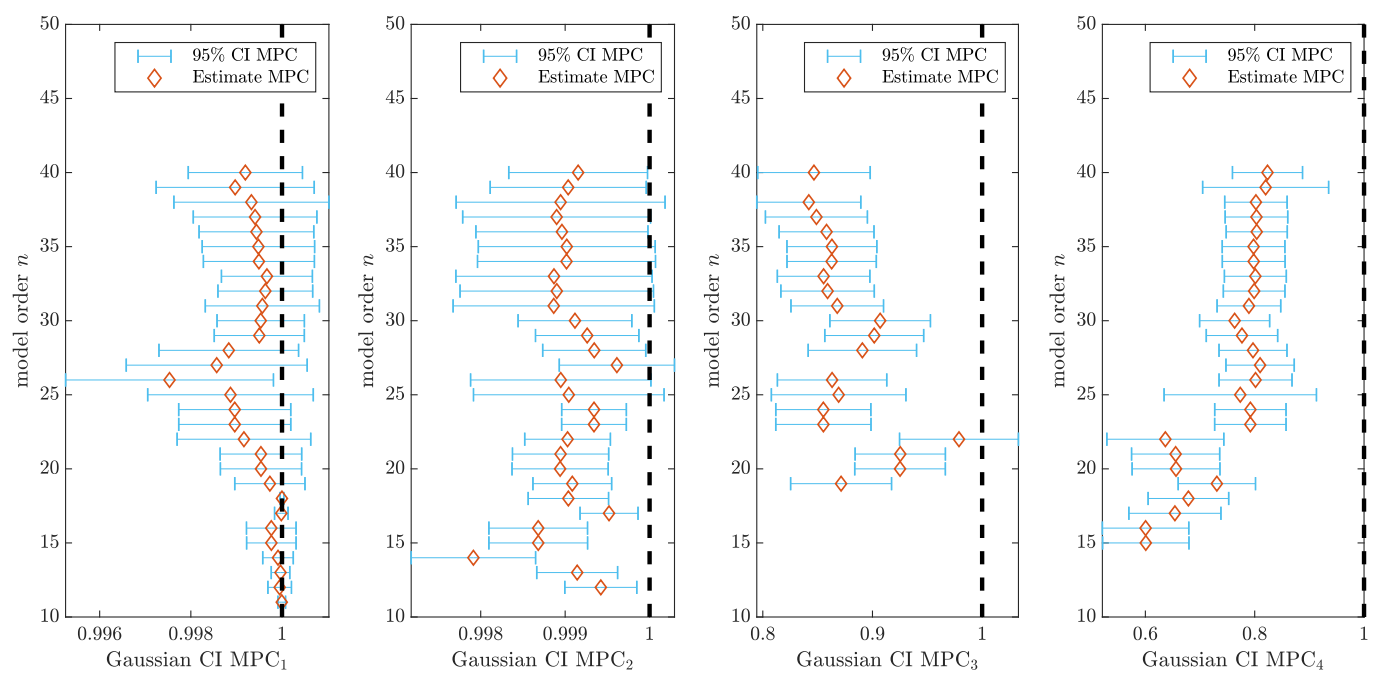

Figure 10: Modal alignments of the MPC with 95\% confidence bounds computed from the mode shapes of the first four modes.

MPC estimate lies within the unit interval.

Based on the analysis above, it can be assumed that the mode shapes corresponding to the second, third and fourth mode are plausibly complex-valued vectors. For the other modes, longer data set could confirm the same assumption. Deciding that the first mode is real-valued would require a more profound analysis for the derivation of confidence interval for real mode shapes.

\section{Discussion}

With the proposed method, confidence intervals are computed based on a Gaussian approximation of the MPC distribution that has been derived for complex mode shapes. The approach assumes that the data length $N$ is large enough for the approximation to be adequate. In particular, it has been illustrated that the Gaussian approximation improves as $N$ increases in Section 3.3, which can also be deduced from the Central Limit Theorem (13) showing a rate of convergence of $1 / \sqrt{N}$.

When applying the method to the uncertainty quantification of the MPC computed from experimental data, it is in general unknown if the underlying mode shape is real-valued or complex-valued. However, the resulting confidence interval is meaningful only for complex mode shapes, where the theoretical MPC is strictly below 1 . Since the MPC distribution is restricted to the unit interval $[0,1]$, meaningful confidence intervals also need to lie within this interval. However, the computed confidence intervals are based on the Gaussian distribution and may overlap 1 in some cases. In such a situation, a problem with the underlying assumptions is revealed: either the data length $N$ has been too short for an adequate Gaussian approximation, or the underlying mode shape is not complex-valued but real-valued. In the former case, a longer data set may yield a better Gaussian approximation with shorter confidence intervals that do not 
contain the value 1 . If for large $N$, the confidence interval still contains 1 , then the considered mode shape could be real-valued. In this case, the theory investigated in the current paper reaches its limits, as condition (11) is not satisfied. Further work should be dedicated to the analysis of the MPC of real mode shapes, which is beyond the scope of this paper.

\section{Conclusion}

In this paper, confidence intervals for the estimate of the Modal Phase Collinearity indicator have been investigated for complex-valued mode shapes. It has been shown that a Gaussian approximation of the distribution of the MPC indicator is adequate for complex mode shapes when the data length is large enough. A computational scheme has been derived to obtain the variance of the MPC estimate, based on the mode shape covariance that is available from modal analysis. This results in a practical method to obtain confidence intervals for the MPC estimate, which is particularly useful to analyze mode shapes whose MPC is high but different than 1. The approach has been validated in Monte Carlo simulations, where the impact of the data length has been analyzed when the MPC is close to 1. Finally, the methodology has been applied to experimental data from a meteorological mast, where the mode shape complexity has been investigated. Future work includes the statistical analysis of the MPC for real-valued mode shapes.

\section{Acknowledgments}

Søren Andreas Nielsen from Universal Foundation A/S is gratefully acknowledged for providing the data set of the meteorological mast for the application part of this paper.

\section{Appendix A. MPC computation}

The eigenvalues $\lambda_{1}^{S}$ and $\lambda_{2}^{S}$ of $S$ in (2) write

$$
\lambda_{1}^{S}=\frac{\left(S_{x x}+S_{y y}\right)+\sqrt{\left(S_{x x}-S_{y y}\right)^{2}+4 S_{x y}^{2}}}{2}, \quad \lambda_{2}^{S}=\frac{\left(S_{x x}+S_{y y}\right)-\sqrt{\left(S_{x x}-S_{y y}\right)^{2}+4 S_{x y}^{2}}}{2} .
$$

Plugging (A.1) into (3) gives directly expression (4). The MPC can be also expressed as a MAC value between a mode shape and its complex conjugate. It writes as follows

$$
\begin{aligned}
\operatorname{MAC}(\varphi, \bar{\varphi}) & =\frac{\varphi^{H} \bar{\varphi} \varphi^{T} \varphi}{\varphi^{H} \varphi \varphi^{T} \bar{\varphi}} \\
& =\frac{\left(\Re(\varphi)^{T}-\mathrm{i} \Im(\varphi)^{T}\right)(\Re(\varphi)-\mathrm{i} \Im(\varphi))\left(\Re(\varphi)^{T}+\mathrm{i} \Im(\varphi)^{T}\right)(\Re(\varphi)+\mathrm{i} \Im(\varphi))}{\left(\Re(\varphi)^{T}-\mathrm{i} \Im(\varphi)^{T}\right)(\Re(\varphi)+\mathrm{i} \Im(\varphi))\left(\Re(\varphi)^{T}+\mathrm{i} \Im(\varphi)^{T}\right)(\Re(\varphi)-\mathrm{i} \Im(\varphi))} \\
& =\frac{\left(S_{x x}-2 \mathrm{i} \Re(\varphi)^{T} \Im(\varphi)-S_{y y}\right)\left(S_{x x}+2 \mathrm{i} \Re(\varphi)^{T} \Im(\varphi)-S_{y y}\right)}{\left(S_{x x}+S_{y y}\right)^{2}} \\
& =\frac{\left(S_{x x}-S_{y y}\right)^{2}+4 S_{x y}^{2}}{\left(S_{x x}+S_{y y}\right)^{2}}=\operatorname{MPC}(\varphi) .
\end{aligned}
$$




\section{Appendix B. Gaussian approximation of MPC distribution}

\section{Appendix B.1. Proof of Property (11)}

For the computation of $\mathcal{J}_{\varphi_{*}}^{\mathrm{MPC}}$ in (8), the partial derivatives (9)-(10) are evaluated in $\varphi_{*}$ and yield

$$
\begin{aligned}
& \frac{\partial \mathrm{MPC}}{\partial \Re(\varphi)}=\frac{4\left(S_{x x}^{*}-S_{y y}^{*}\right) \Re\left(\varphi_{*}\right)^{T}+8 S_{x y}^{*} \Im\left(\varphi_{*}\right)^{T}-4 \operatorname{MPC}\left(\varphi_{*}\right) c_{*} \Re\left(\varphi_{*}\right)^{T}}{\left(c_{*}\right)^{2}}, \\
& \frac{\partial \mathrm{MPC}}{\partial \Im(\varphi)}=\frac{4\left(S_{y y}^{*}-S_{x x}^{*}\right) \Im\left(\varphi_{*}\right)^{T}+8 S_{x y}^{*} \Re\left(\varphi_{*}\right)^{T}-4 \operatorname{MPC}\left(\varphi_{*}\right) c_{*} \Im\left(\varphi_{*}\right)^{T}}{\left(c_{*}\right)^{2}},
\end{aligned}
$$

where the respective scalars $S_{x x}^{*}, S_{y y}^{*}, S_{x y}^{*}$ and $c_{*}$ correspond to $S_{x x}, S_{y y}, S_{x y}$ and $c$ computed with $\varphi_{*}$.

First it is proved that $\operatorname{MPC}\left(\varphi_{*}\right) \in\{0,1\} \Rightarrow \mathcal{J}_{\varphi_{*}}^{\mathrm{MPC}}=0$. First consider the case $\operatorname{MPC}\left(\varphi_{*}\right)=1$. From the definition of the MPC in (4) it follows

$$
\begin{aligned}
& \left(S_{x x}^{*}-S_{y y}^{*}\right)^{2}+4\left(S_{x y}^{*}\right)^{2}=\left(S_{x x}^{*}+S_{y y}^{*}\right)^{2}, \\
& \Rightarrow 4 S_{x x}^{*} S_{y y}^{*}=4 S_{x y}^{* 2}, \\
& \Rightarrow\left\|\Re\left(\varphi_{*}\right)\right\|^{2}\left\|\Im\left(\varphi_{*}\right)\right\|^{2}=\left\|\Re\left(\varphi_{*}\right)\right\|^{2}\left\|\Im\left(\varphi_{*}\right)\right\|^{2} \cos ^{2}\left(\Re\left(\varphi_{*}\right), \Im\left(\varphi_{*}\right)\right), \\
& \Rightarrow \cos ^{2}\left(\Re\left(\varphi_{*}\right), \Im\left(\varphi_{*}\right)\right)=1 .
\end{aligned}
$$

Hence, the angle between the real and imaginary part of $\varphi_{*}$ is 0 when the MPC is 1 . Then, assuming without loss of generality that the real part is non-zero, the imaginary part must be a multiple of the real part with $a \cdot \Re\left(\varphi_{*}\right)=\Im\left(\varphi_{*}\right)$, where $a \in \mathbb{R}$ is a scalar. Consequently, (B.1) and (B.2) write as

$$
\begin{aligned}
& \frac{\partial \mathrm{MPC}}{\partial \Re(\varphi)}=\frac{4\left(S_{x x}^{*}-a^{2} S_{x x}^{*}\right) \Re\left(\varphi_{*}\right)^{T}+8 a^{2} S_{x x}^{*} \Re\left(\varphi_{*}\right)^{T}-4\left(S_{x x}^{*}+a^{2} S_{x x}^{*}\right) \Re\left(\varphi_{*}\right)^{T}}{c_{*}^{2}}=0, \\
& \frac{\partial \mathrm{MPC}}{\partial \Im(\varphi)}=\frac{4\left(a^{2} S_{x x}^{*}-S_{x x}^{*}\right) a \Re\left(\varphi_{*}\right)^{T}+8 a S_{x x}^{*} \Re\left(\varphi_{*}\right)^{T}-4\left(S_{x x}^{*}+a^{2} S_{x x}^{*}\right) a \Re\left(\varphi_{*}\right)^{T}}{c_{*}^{2}}=0 .
\end{aligned}
$$

Similarly, for the case $\operatorname{MPC}\left(\varphi_{*}\right)=0$ it follows $\left(S_{x x}^{*}-S_{y y}^{*}\right)^{2}+4\left(S_{x y}^{*}\right)^{2}=0$ from (4), thus $S_{x x}^{*}=S_{y y}^{*}$ and $S_{x y}^{*}=0$. Plugging this into (B.1) and (B.2) yields $\frac{\partial \mathrm{MPC}}{\partial \Re(\varphi)}=0$ and $\frac{\partial \mathrm{MPC}}{\partial \Im(\varphi)}=0$, which concludes the first part of the proof.

Second it is proved that $\mathcal{J}_{\varphi_{*}}^{\mathrm{MPC}}=0 \Rightarrow \operatorname{MPC}\left(\varphi_{*}\right) \in\{0,1\}$. Since (B.1) is zero, this yields

$$
4\left(S_{x x}^{*}-S_{y y}^{*}\right) \Re\left(\varphi_{*}\right)^{T}+8 S_{x y}^{*} \Im\left(\varphi_{*}\right)^{T}-4 \operatorname{MPC}\left(\varphi_{*}\right) \Re\left(\varphi_{*}\right)^{T}\left(S_{x x}^{*}+S_{y y}^{*}\right)=0 .
$$

Consider two cases, namely $S_{x y}^{*} \neq 0$ and $S_{x y}^{*}=0$. In the first case, multiplying (B.3) from the right side with $\Im\left(\varphi_{*}\right)$ (which is $\neq 0$ since $S_{x y}^{*} \neq 0$ ) yields

$$
\begin{aligned}
4\left(S_{x x}^{*}-S_{y y}^{*}\right) S_{x y}^{*}+8 S_{x y}^{*} S_{y y}^{*} & =4 S_{x y}^{*} \operatorname{MPC}\left(\varphi_{*}\right)\left(S_{x x}^{*}+S_{y y}^{*}\right) \\
4\left(S_{x x}^{*}+S_{y y}^{*}\right) & =4 \operatorname{MPC}\left(\varphi_{*}\right)\left(S_{x x}^{*}+S_{y y}^{*}\right) \Rightarrow \operatorname{MPC}\left(\varphi_{*}\right)=1 .
\end{aligned}
$$


In the second case when $S_{x y}^{*}=0$, distinguish furthermore the cases $S_{x x}^{*}=S_{y y}^{*}$ and $S_{x x}^{*} \neq S_{y y}^{*}$. When $S_{x x}^{*}=S_{y y}^{*}$, then in particular $S_{x x}^{*} \neq 0$ and $\Re\left(\varphi_{*}\right) \neq 0$, otherwise the mode shape would be 0. From (B.3) it follows then directly $\operatorname{MPC}\left(\varphi_{*}\right)=0$. When $S_{x x}^{*} \neq S_{y y}^{*}$, then since both terms (B.1) and (B.2) are 0 it holds also $\frac{\partial \mathrm{MPC}}{\partial \Re(\varphi)} \cdot \Re\left(\varphi_{*}\right)-\frac{\partial \mathrm{MPC}}{\partial \Im(\varphi)} \cdot \Im\left(\varphi_{*}\right)=0$ and thus

$$
4\left(S_{x x}^{*}-S_{y y}^{*}\right)\left(S_{x x}^{*}+S_{y y}^{*}\right)-4 \operatorname{MPC}\left(\varphi_{*}\right)\left(S_{x x}^{*}+S_{y y}^{*}\right)\left(S_{x x}^{*}-S_{y y}^{*}\right)=0 \Rightarrow \operatorname{MPC}\left(\varphi_{*}\right)=1 .
$$

Thus, all the possible cases for the second part of the proof lead to $\operatorname{MPC}\left(\varphi_{*}\right)=0$ or $\operatorname{MPC}\left(\varphi_{*}\right)=1$, which concludes the proof.

\section{Appendix B.2. Proof of Property (13)}

In order to apply the first-order delta method, it is required that $\sigma_{\mathrm{MPC}}^{2}=\mathcal{J}_{\varphi_{*}}^{\mathrm{MPC}} \Sigma_{\varphi_{*}}\left(\mathcal{J}_{\varphi_{*}}^{\mathrm{MPC}}\right)^{T} \neq 0$. Since $\mathcal{J}_{\varphi_{*}}^{\mathrm{MPC}} \neq 0$ due to property (11), it remains to show that $\left(\mathcal{J}_{\varphi_{*}}^{\mathrm{MPC}}\right)^{T}$ is not in the null space of $\Sigma_{\varphi_{*}}$. Since the mode shape estimate is defined up to a constant, it may be the direct unnormalized result of the eigenvalue decomposition, or a normalization may be applied depending on the identification procedure, which determines the properties of $\Sigma_{\varphi_{*}}$. Here it is assumed that the identified mode shape is either unnormalized, or that one of two widely used normalization schemes has been applied either with respect to one mode shape component (called Normalization 1 [2]), or additionally with respect to the norm of the mode shape (Normalization $2[9])$.

When no mode shape normalization has been applied, $\Sigma_{\varphi_{*}}$ is full rank, assuming no dependencies between the estimates of the mode shape components. Hence there is no null space and thus $\sigma_{\mathrm{MPC}}^{2} \neq 0$.

When mode shape normalization has been applied, denote $\tilde{\varphi}$ the unnormalized mode shape estimate. For the considered normalization schemes it holds:

Normalization 1: The normalized mode shape is $\hat{\varphi}=\tilde{\varphi} / \tilde{\varphi}_{k}$ for some component $k$ with asymptotically $\tilde{\varphi}_{k} \neq 0$. Its asymptotic covariance is given by $\Sigma_{\varphi_{*}}=\mathcal{J}_{\hat{\varphi}, \tilde{\varphi}} \Sigma_{\tilde{\varphi}} \mathcal{J}_{\hat{\varphi}, \tilde{\varphi}}^{T}$, where the estimate of $\mathcal{J}_{\hat{\varphi}, \tilde{\varphi}}$ yields [2, 9]

$$
\widehat{\mathcal{J}}_{\hat{\varphi}, \tilde{\varphi}}=\left[\begin{array}{cc}
\Re\left(\widehat{\mathcal{J}}_{\hat{\varphi}, \tilde{\varphi}}^{c}\right) & -\Im\left(\widehat{\mathcal{J}}_{\hat{\varphi}, \tilde{\varphi}}^{c}\right) \\
\Im\left(\widehat{\mathcal{J}}_{\hat{\varphi}, \tilde{\varphi}}^{c}\right) & \Re\left(\widehat{\mathcal{J}}_{\hat{\varphi}, \tilde{\varphi}}^{c}\right)
\end{array}\right], \quad \text { where } \quad \widehat{\mathcal{J}}_{\hat{\varphi}, \tilde{\varphi}}^{c}=\frac{1}{\tilde{\varphi}_{k}}\left(I_{r}-\frac{1}{\tilde{\varphi}_{k}} \tilde{\varphi} e_{k}^{T}\right)
$$

and $e_{k} \in \mathbb{R}^{r}$ is the unit vector with entry 1 at component $k$. Notice $\operatorname{rank}\left(\Sigma_{\varphi_{*}}\right)=\operatorname{rank}\left(\mathcal{J}_{\hat{\varphi}, \tilde{\varphi}}\right)$. Since $\mathcal{J}_{\hat{\varphi}, \tilde{\varphi}}^{c}$ is the sum of the identity matrix and a dyadic product, $\operatorname{rank}\left(\mathcal{J}_{\hat{\varphi}, \tilde{\varphi}}\right) \geq r-1$. It holds $e_{k}^{T} \mathcal{J}_{\hat{\varphi}, \tilde{\varphi}}^{c}=0$, thus $\operatorname{rank}\left(\mathcal{J}_{\hat{\varphi}, \tilde{\varphi}}^{c}\right)=r-1$ and $\operatorname{rank}\left(\Sigma_{\varphi_{*}}\right)=\operatorname{rank}\left(\mathcal{J}_{\hat{\varphi}, \tilde{\varphi}}\right)=2 r-2$. The two null space vectors $n_{1}, n_{2} \in \mathbb{R}^{2 r}$ of $\Sigma_{\varphi_{*}}$ follow as $n_{1}^{T}=\left[\begin{array}{ll}e_{k}^{T} & 0\end{array}\right], n_{2}^{T}=\left[\begin{array}{ll}0 & e_{k}^{T}\end{array}\right]$. To show $\sigma_{\mathrm{MPC}}^{2} \neq 0$, it needs to be shown that there exist no scalars $a_{1}, a_{2} \in \mathbb{R}$ such that

$$
\mathcal{J}_{\varphi_{*}}^{\mathrm{MPC}}=a_{1} n_{1}^{T}+a_{2} n_{2}^{T} .
$$

Assume the contrary, namely that (B.5) actually holds. Then, a contradiction appears by multiplying (B.5)

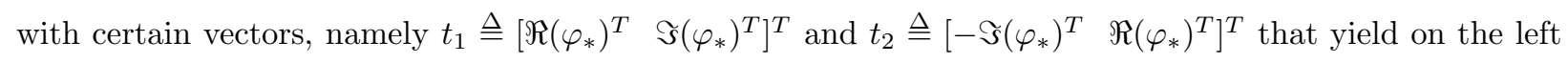


hand side $\mathcal{J}_{\varphi_{*}}^{\mathrm{MPC}} t_{1}=0$ and $\mathcal{J}_{\varphi_{*}}^{\mathrm{MPC}} t_{2}=0$ which follows immediately from (B.1), (B.2) and (4). Since $\varphi_{k}=1$, on the right hand side it follows $n_{1}^{T} t_{1}=1$ and $n_{2}^{T} t_{1}=0$ thus $a_{1}=0$, and $n_{1}^{T} t_{2}=0$ and $n_{2}^{T} t_{2}=1$ thus $a_{2}=0$. Then $\mathcal{J}_{\varphi_{*}}^{\mathrm{MPC}}=0$ in (B.5), which is a contradiction to $\mathcal{J}_{\varphi_{*}}^{\mathrm{MPC}} \neq 0$. Hence, (B.5) cannot hold, thus $\left(\mathcal{J}_{\varphi_{*}}^{\mathrm{MPC}}\right)^{T}$ is not in the null space of $\Sigma_{\varphi_{*}}$ and $\sigma_{\mathrm{MPC}}^{2} \neq 0$.

Normalization 2: The normalized mode shape is $\hat{\varphi}=\breve{\varphi} /\|\breve{\varphi}\|$ with $\breve{\varphi}=\tilde{\varphi} / \tilde{\varphi}_{k}$. A first-order perturbation yields $\Delta \hat{\varphi}=-\frac{\breve{\varphi}}{\|\breve{\varphi}\|^{3}} \Re\left(\breve{\varphi}^{H} \Delta \breve{\varphi}\right)+\frac{1}{\|\breve{\varphi}\|} \Delta \breve{\varphi}$, thus

$$
\Delta\left[\begin{array}{l}
\Re(\hat{\varphi}) \\
\Im(\hat{\varphi})
\end{array}\right]=\widehat{\mathcal{J}}_{\hat{\varphi}, \breve{\varphi}} \Delta\left[\begin{array}{l}
\Re(\breve{\varphi}) \\
\Im(\breve{\varphi})
\end{array}\right] \text { with } \widehat{\mathcal{J}}_{\hat{\varphi}, \breve{\varphi}}=-\frac{1}{\|\breve{\varphi}\|^{3}}\left[\begin{array}{l}
\Re(\breve{\varphi}) \\
\Im(\breve{\varphi})
\end{array}\right]\left[\begin{array}{c}
\Re(\breve{\varphi}) \\
\Im(\breve{\varphi})
\end{array}\right]^{T}+\frac{1}{\|\breve{\varphi}\|} I_{2 r},
$$

and the asymptotic covariance of the normalized mode shape follows as $\Sigma_{\varphi_{*}}=\mathcal{J}_{\hat{\varphi}, \breve{\varphi}} \mathcal{J}_{\breve{\varphi}, \breve{\varphi}} \Sigma_{\tilde{\varphi}} \mathcal{J}_{\hat{\varphi}, \breve{\varphi}}^{T} \mathcal{J}_{\hat{\varphi}, \breve{\varphi}}^{T}$ with $\mathcal{J}_{\breve{\varphi}, \tilde{\varphi}}$ as in (B.4). Notice $\operatorname{rank}\left(\Sigma_{\varphi_{*}}\right)=\operatorname{rank}\left(\mathcal{J}_{\hat{\varphi}, \breve{\varphi}} \mathcal{J}_{\breve{\varphi}, \tilde{\varphi}}\right)$. Since $\mathcal{J}_{\hat{\varphi}, \breve{\varphi}}$ is the sum of the identity matrix and a dyadic product, $\operatorname{rank}\left(\mathcal{J}_{\hat{\varphi}, \breve{\varphi}}\right) \geq 2 r-1$. It holds $\left[\Re(\breve{\varphi})^{T} \Im(\breve{\varphi})^{T}\right] \mathcal{J}_{\hat{\varphi}, \breve{\varphi}}=0$, thus $\operatorname{rank}\left(\mathcal{J}_{\hat{\varphi}, \breve{\varphi}}\right)=2 r-1$. Since

$$
\left[\begin{array}{ll}
\Re(\breve{\varphi})^{T} & \Im(\breve{\varphi})^{T}
\end{array}\right]\left[\begin{array}{c}
e_{k} \\
0
\end{array}\right] \neq 0 \text { but }\left[\Re(\breve{\varphi})^{T} \quad \Im(\breve{\varphi})^{T}\right] \mathcal{J}_{\hat{\varphi}, \breve{\varphi}}=0
$$

the left null space vector $\left[\begin{array}{ll}e_{k}^{T} & 0\end{array}\right]^{T}$ of $\mathcal{J}_{\breve{\varphi}, \tilde{\varphi}}$ cannot be in the image of $\mathcal{J}_{\hat{\varphi}, \breve{\varphi}}$ and it follows $\operatorname{rank}\left(\Sigma_{\varphi_{*}}\right)=$ $\operatorname{rank}\left(\mathcal{J}_{\hat{\varphi}, \breve{\varphi}} \mathcal{J}_{\breve{\varphi}, \tilde{\varphi}}\right)=2 r-2$.

Thus, there are two null space vectors $n_{1}, n_{2} \in \mathbb{R}^{2 r}$ of $\Sigma_{\varphi_{*}}$ that follow as $n_{1}^{T}=\left[\Re(\breve{\varphi})^{T} \Im(\breve{\varphi})^{T}\right]$, and $n_{2}^{T}=\left[\begin{array}{ll}0 & e_{k}^{T}\end{array}\right]$ since $\left[\begin{array}{ll}0 & e_{k}^{T}\end{array}\right] \mathcal{J}_{\hat{\varphi}, \breve{\varphi}}=\frac{1}{\|\breve{\varphi}\|}\left[\begin{array}{ll}0 & e_{k}^{T}\end{array}\right]$ which is in the left null space of $\mathcal{J}_{\breve{\varphi}, \tilde{\varphi}}$. To show $\sigma_{\mathrm{MPC}}^{2} \neq 0$, it needs to be shown that there exist no scalars $a_{1}, a_{2} \in \mathbb{R}$ satisfying (B.5) analogously to the first normalization, by multiplying $t_{1}$ and $t_{2}$ to (B.5). Since $\Im\left(\varphi_{k}\right)=0$, it follows $n_{1}^{T} t_{1}=1$ and $n_{2}^{T} t_{1}=0$ thus $a_{1}=0$, and $n_{1}^{T} t_{2}=0$ and $n_{2}^{T} t_{2}=\Re\left(\varphi_{k}\right) \neq 0$ thus $a_{2}=0$. Hence, (B.5) cannot hold, thus $\left(\mathcal{J}_{\varphi_{*}}^{\mathrm{MPC}}\right)^{T}$ is not in the null space of $\Sigma_{\varphi_{*}}$ and $\sigma_{\mathrm{MPC}}^{2} \neq 0$.

\section{References}

[1] E. Reynders, System identification methods for (operational) modal analysis: review and comparison, Archives of Computational Methods in Engineering 19 (1) (2012) 51-124.

[2] E. Reynders, R. Pintelon, G. De Roeck, Uncertainty bounds on modal parameters obtained from stochastic subspace identification, Mechanical Systems and Signal Processing 22 (4) (2008) 948-969.

[3] R. Pintelon, P. Guillaume, J. Schoukens, Uncertainty calculation in (operational) modal analysis, Mechanical Systems and Signal Processing 21 (6) (2007) 2359-2373.

[4] M. Döhler, L. Mevel, Efficient multi-order uncertainty computation for stochastic subspace identification, Mechanical Systems and Signal Processing 38 (2) (2013) 346-366.

[5] M. Döhler, X.-B. Lam, L. Mevel, Multi-order covariance computation for estimates in stochastic subspace identification using QR decompositions, IFAC Proceedings Volumes 47 (3) (2014) 9498-9503, 19th IFAC World Congress.

[6] M. Döhler, F. Hille, L. Mevel, W. Rücker, Structural health monitoring with statistical methods during progressive damage test of S101 Bridge, Engineering Structures 69 (2014) 183-193. 
[7] M. Döhler, P. Andersen, L. Mevel, Variance computation of modal parameter estimates from UPC subspace identification, in: Proceedings of the 7th International Operational Modal Analysis Conference, Ingolstadt, Germany, 2017.

[8] E. Reynders, K. Maes, G. Lombaert, G. De Roeck, Uncertainty quantification in operational modal analysis with stochastic subspace identification: Validation and applications, Mechanical Systems and Signal Processing 66-67 (2016) 13-30.

[9] M. Döhler, X.-B. Lam, L. Mevel, Uncertainty quantification for modal parameters from stochastic subspace identification on multi-setup measurements, Mechanical Systems and Signal Processing 36 (2013) 562-581.

[10] P. Mellinger, M. Döhler, L. Mevel, Variance estimation of modal parameters from output-only and input/output subspacebased system identification, Journal of Sound and Vibration 379 (2016) 1-27.

[11] T. K. Caughey, M. E. J. O'Kelly, Classical normal modes in damped linear dynamic systems, Journal of Applied Mechanics 32 (3) (1965) 583-588.

[12] S. Adhiakri, Classical normal modes in nonviscously damped linear systems, AIAA Journal 39 (5) (2001) $978-980$.

[13] C. Hoen, An engineering interpretation of the complex eigensolution of linear dynamic systems, in: Proceedings of the XXIII IMAC Conference, 2005.

[14] S. Adhikari, Optimal complex modes and an index of damping non-proportionality, Mechanical Systems and Signal Processing 18 (1) (2004) $1-27$.

[15] J. T. Sawicki, G. Genta, Modal uncoupling of damped gyroscopic systems, Journal of Sound and Vibration 244 (3) (2001) $431-451$.

[16] E. Swanson, C. Powell, S. Weissman, A practical review of rotating machinery critical speeds and modes, Sound and Vibration 39 (2005) 10-17.

[17] X. Chen, M. Matsumoto, A. Kareem, Aerodynamic coupling effects on flutter and buffeting of bridges, Journal of Engineering Mechanics 126 (1) (2000) 17-26.

[18] D. Bernal, Complex eigenvector scaling from mass perturbations, Mechanical Systems and Signal Processing 45 (1) (2014) 80-90.

[19] P. Olsen, A. Skafte, J. Hansen, M. Hovgaard, R. Brincker, C. Ventura, Identification of systems with complex modes using OMA, in: Proceedings of the 5th IOMAC International Operational Modal Analysis Conference, 2013.

[20] R. Pappa, K. B. Elliott, A. Schenk, Consistent-mode indicator for the eigensystem realization algorithm, Journal of Guidance, Control, and Dynamics 16 (5) (1993) 852-858.

[21] Structural Vibration Solutions A/S, ARTeMIS Modal Pro 6.0, http://www.svibs.com (2018).

[22] M. Vigs $\varnothing$, T. Kabel, M. Tarp $\varnothing$, R. Brincker, C. Georgakis, Operational modal analysis and fluid-structure interaction, in: Proceedings of the 28th International Conference on Noise and Vibration Engineering (ISMA), Leuven, Belgium, 2018.

[23] G. Casella, R. L. Berger, Statistical inference, 2nd Edition, Duxbury Press, Pacific Grove, CA, USA, 2001.

[24] P. Vacher, B. Jacquier, A. Bucharles, Extensions of the MAC criterion to complex modes, in: Proceedings of the 24th International Conference on Noise and Vibration Engineering (ISMA), Leuven, Belgium, 2010.

[25] J.-N. Juang, R. S. Pappa, An eigensystem realization algorithm for modal parameter identification and model reduction, Journal of Guidance, Control, and Dynamics 8 (5) (1985) 620-627.

[26] R. J. Allemang, The modal assurance criterion - twenty years of use and abuse, Sound and Vibration Magazine (2003) $14-21$.

[27] B. Peeters, G. De Roeck, Reference-based stochastic subspace identification for output-only modal analysis, Mechanical Systems and Signal Processing 13 (6) (1999) 855-878.

[28] M. Döhler, L. Mevel, F. Hille, Subspace-based damage detection under changes in the ambient excitation statistics, Mechanical Systems and Signal Processing 45 (1) (2014) 207-224.

[29] M. Bhuyan, G. Gautier, N. Le Touz, M. Döhler, F. Hille, J. Dumoulin, L. Mevel, Vibration-based damage localization with load vectors under temperature changes, Structural Control and Health Monitoring 26 (11) (2019) e2439. 
[30] E. Viefhues, M. Döhler, Q. Zhang, F. Hille, L. Mevel, Subspace-based damage detection with rejection of the temperature effect and uncertainty in the reference, in: Proc. 8th International Operational Modal Analysis Conference, Copenhagen, Denmark, 2019.

[31] D. Bauer, M. Deistler, W. Scherrer, Consistency and asymptotic normality of some subspace algorithms for systems without observed inputs, Automatica 35 (7) (1999) 1243-1254.

[32] K. Knight, Mathematical Statistics, 1st Edition, Chapman and Hall/CRC, 2000.

[33] S. Greś, M. Fejerskov, L. Ibsen, L. Damkilde, Experimental damping assessment of a full scale offshore mono bucket foundation, in: P. Sas, D. Moens, A. van de Walle (Eds.), Proceedings of ISMA2016, KU Leuven, Department of Mechanical Engineering, PMA, 2016, pp. 4045-4054, the International Conference on Noise and Vibration Engineering, ISMA. 